\title{
A NOTE ON THE QUILLEN-LICHTENBAUM CONJECTURE AND THE ARITHMETIC OF SQUARE RINGS
}

\author{
G.Banaszak, W.Gajda, P.Krasoń and P.Zelewski
}

Abstract. In this paper we consider the Quillen-Lichtenbaum conjecture for number fields using description of the higher $K$-theory in terms of $S K_{1}$ groups.

\section{INTRODUCTION}

Let $F$ be a number field and let $n$ be a non-negative integer. Fix an odd prime $l$. We define the wild kernel $W K_{n}(F)$ as the kernel of the localization map in $K$-theory of $F$ :

$$
\lambda_{n}: K_{n}(F) \rightarrow \prod_{v} K_{n}\left(F_{v}\right)
$$

The symbol $\prod_{v}$ denotes the usual direct product. When $n$ is even, the image of $\lambda_{n}$ is contained in $\bigoplus_{v} K_{n}\left(F_{v}\right)$. However for $n$ odd, the image is not contained in the direct sum, cf. diagram (3.4) below and [AB, Theorem 1]. The group $W K_{n}(F)$ is interesting because we have:

Proposition A. [Prop. 5.1.] For each $n \geq 0$

$$
W K_{n}(F) \subseteq K_{n}(\mathcal{O})_{t o r}
$$

where $\mathcal{O}$ denotes the ring of integers of $F$. In particular, the group $W K_{n}(F)$ is finite.

By $[\mathrm{B}$, theorem 2] there is the following long exact sequence.

$$
0 \rightarrow K_{2 n}^{w}(\mathcal{O})_{l} \rightarrow K_{2 n}(F)_{l} \rightarrow \bigoplus_{v} W^{n}\left(F_{v}\right) \rightarrow W^{n}(F) \rightarrow 0
$$

where $v$ runs over finite places of $F$. In [B] the group $K_{2 n}^{w}(\mathcal{O})_{l}$ was called the $l$-part of the wild kernel for $K_{2 n}(F)$. We expect that the groups $W K_{2 n}(F)_{l}$ and $K_{2 n}^{w}(\mathcal{O})_{l}$ coincide. It is unknown in the moment, since we do not know the $K$-theory with coefficients in $\mathbb{Z} / l^{k}$ of a local field in the case when $l$ is divisible by the residue characteristic, except for the case of $\mathbb{Q}_{l}$ which follows by results of Bökstedt and Madsen [BM].

1991 Mathematics Subject Classification. Primary 19F27; Secondary 19Fxx.

This work has been sponsored in part by a KBN grant 2P03A 00511.

Typeset by $\mathcal{A} \mathcal{M} \mathcal{S}-\mathrm{T}_{\mathrm{E}} \mathrm{X}$ 
We propose the following conjecture.

Conjecture B. For all number fields $F$ and for all $n \geq 0$ we have:

$$
W K_{n}(F)_{l}=\left[\operatorname{div} K_{n}(F)\right]_{l}
$$

where div $K_{n}(F)$ is the group of divisible elements in $K_{n}(F)$.

In section 5 of the paper we give the following condition for equivalence of the above conjecture with the Quillen-Lichtenbaum conjecture for the number field $F$ [DF, p. 273].

Theorem C. [Th. 5.2.]

(a) Assume that the following maps:

$$
K_{2 m}\left(F_{v}\right)_{l} \rightarrow K_{2 m}^{e t}\left(F_{v}\right)_{l}
$$

are isomorphisms for all $v$ which divide $l$ and for all $m>0$. Then the Quillen-Lichtenbaum conjecture for $F$ is equivalent to the conjecture $B$ for all indices $n>1$.

(b) If $F=\mathbb{Q}$, then the Quillen-Lichtenbaum conjecture and conjecture $B$ are equivalent.

Theorem D. [Th. 5.5.] The conjecture $B$ holds true for all number fields $F$ and for $0 \leq n \leq 3$. In addition, it holds true for $n=4$ and $F=\mathbb{Q}$.

For another reformulation of the Quillen-Lichtenbaum conjecture see [BZ1, p. 390].

By [BZ2, theorem, p.161] and a result of Dayton and Weibel [DW, Corollary 5.3] we get the following exact sequence involving the wild kernel (see Section 6).

$$
0 \rightarrow W K_{n}(F) \rightarrow S K_{1}\left(F_{n}\right) \rightarrow \prod_{v} S K_{1}\left(F_{v n}^{h}\right)
$$

In this way we reformulate the conjecture B in terms of elementary linear algebra, number fields and algebraic properties of the square rings $F_{n}$. In the last section of the paper we investigate $W K_{n}(F)$ using the description of the higher $K$-theory in terms of $S K_{1}$.

\section{Notation}

(1) We fix an odd rational prime $l$.

(2) Let $F$ be a number field and let $\mathcal{O}$ be its ring of integers.

(3) Let $v$ be a finite place of $\mathcal{O}$ and let $k_{v}$ be the residue field for $v$.

(4) Put $F_{v}$ to be the completion of $F$ at $v$ and $\mathcal{O}_{v}$ to be the ring of integers of $F_{v}$.

(5) Put $\mathcal{O}_{v}^{h}$ to be the henselization of $\mathcal{O}_{F}$ at $v$ and $F_{v}^{h}$ to be the quotient field of $\mathcal{O}_{v}^{h}$. 
(6) For a commutative ring with identity $R$ and for $n>0$ we denote by $R_{n}$ the square ring:

$$
R\left[t_{1}, t_{2}, \ldots, t_{n}\right] /\left(\left(t_{1}^{2}-t_{1}\right) \ldots\left(t_{n}^{2}-t_{n}\right)\right) .
$$

(7) Let $K_{*}(-)\left(K_{*}\left(., \mathbb{Z} / l^{k}\right)\right.$ resp.) denote the Quillen $K$-theory ( $K$-theory with coefficients defined by Browder and Karoubi, resp.).

(8) Let $K_{*}^{e t}().\left(K_{*}^{e t}\left(., \mathbb{Z} / l^{k}\right)\right.$, resp.) denote the etale $K$-theory (etale $K$-theory with finite coefficients, resp.) defined by Dwyer and Friedlander.

(9) For an abelian group $G$ we denote by $G_{l}$ its $l$-torsion part and by $G\left[l^{k}\right]$ the elements of $G$ with orders dividing $l^{k}$.

(10) $\operatorname{div} A=\bigcap_{r>1} A^{r}$ denotes the subgroup of infinitely divisible elements inside an abelian group $A$.

\section{ACKNOWLEDGMENTS}

The first three authors thank the Max-Planck-Institut für Mathematik in Bonn and Fakultät für Mathematik Bielefeld Universität in Germany where parts of this work were done. The second author was supported by a research fellowship of Alexander von Humboldt Foundation at Bielefeld Universität when this paper was completed. He thanks both institutions for support and hospitality. The authors thank the referee for careful reading of the manuscript and useful remarks.

\section{INTRODUCTORY COMPUTATIONS}

The computations below follow easily from well-known results but we include some detailed explanations for the convenience of the reader. By [Su1] we have the following isomorphism for all $l$ different from the residue characteristic of $k_{v}$, for all $j>0$ and all nonnegative $n$.

$$
K_{n}\left(\mathcal{O}_{v} ; \mathbb{Z} / l^{j}\right) \rightarrow K_{n}\left(k_{v} ; \mathbb{Z} / l^{j}\right)
$$

Using the Bockstein exact sequences for $K$-theory with finite coefficients we get the following diagram.

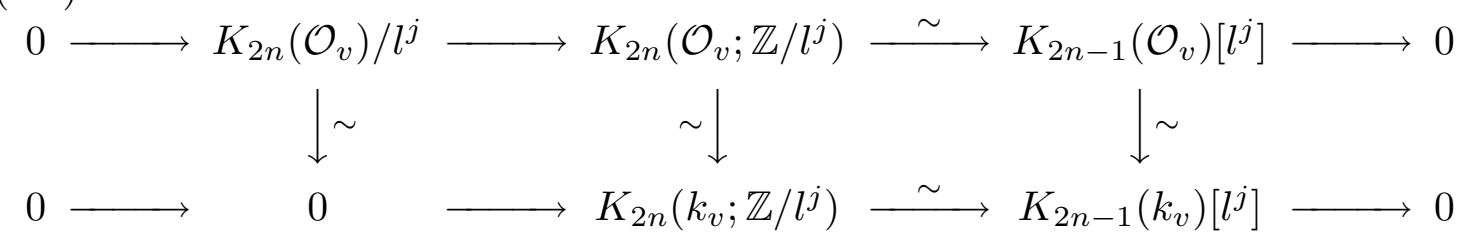

The diagram (2.1) shows that the group $K_{2 n}\left(\mathcal{O}_{v}\right)$ is $l$-divisible for all $n>0$. Consider also the following commutative diagram:

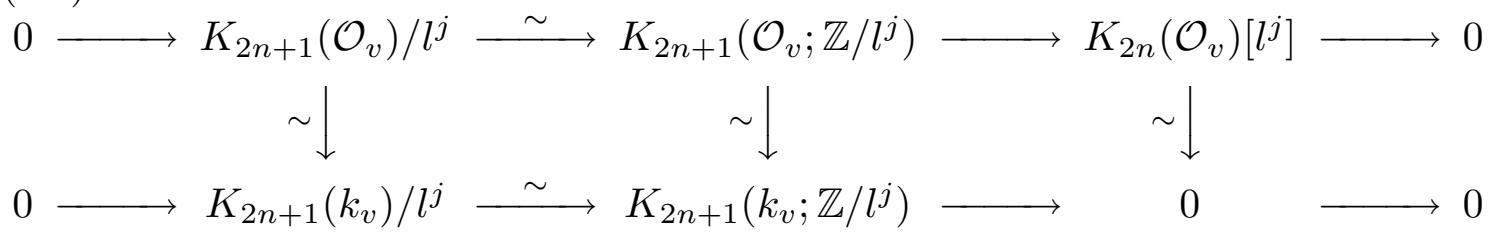


Consider $n>0$. From the diagram (2.1) we see that if $j$ is big enough, then we have:

$$
K_{2 n-1}\left(\mathcal{O}_{v}\right)_{l}=K_{2 n-1}\left(k_{v}\right)_{l}=\text { finite }
$$

Hence, the $l$-torsion part of the group $K_{2 n-1}\left(\mathcal{O}_{v}\right)_{l}$ can not be divisible. In addition, we see that:

$$
K_{2 n-1}\left(k_{v}\right)_{l}=K_{2 n-1}\left(k_{v}\right) / l^{j}
$$

so the left vertical arrow in the diagram (2.2) is surjective for $j$ big enough. This implies that the right vertical arrow in $(2.2)$ is an isomorphism. Hence, $K_{2 n}\left(\mathcal{O}_{v}\right)_{l}=$ 0 . This shows that the left square in the diagram (2.2) consists of isomorphisms. So the group $K_{2 n}\left(\mathcal{O}_{v}\right)$ is uniquely $l$-divisible. Let us consider the following localization exact sequence.

$$
0 \rightarrow K_{2 n}\left(\mathcal{O}_{v}\right) \rightarrow K_{2 n}\left(F_{v}\right) \rightarrow K_{2 n-1}\left(k_{v}\right) \rightarrow 0
$$

We know by [BZ1, p. 386] that:

$$
K_{2 n}\left(F_{v}\right)=K_{2 n}\left(F_{v}\right)_{l} \bigoplus l^{j_{0}} K_{2 n}\left(F_{v}\right)
$$

and the group $l^{j_{0}} K_{2 n}\left(F_{v}\right)$ is uniquely $l$-divisible. We can also write:

$$
K_{2 n-1}\left(k_{v}\right)=K_{2 n-1}\left(k_{v}\right)_{l} \bigoplus(\text { non } l \text {-part })
$$

Hence, we observe that the boundary map in the localization sequence (2.4) sends the subgroup $K_{2 n}\left(F_{v}\right)_{l} \oplus 0$ of $K_{2 n}\left(F_{v}\right)$ into:

$$
K_{2 n-1}\left(k_{v}\right)_{l} \bigoplus 0 \subseteq K_{2 n-1}\left(k_{v}\right)
$$

and it sends $0 \bigoplus l^{j_{0}} K_{2 n}\left(F_{v}\right)$ into:

$$
0 \bigoplus(\text { non } l \text { part }) \subseteq K_{2 n-1}\left(k_{v}\right)
$$

It follows that the map:

$$
K_{2 n}\left(F_{v}\right)_{l} \stackrel{\sim}{\longrightarrow} K_{2 n-1}\left(k_{v}\right)_{l} .
$$

is surjective. We know that this map is injective because $K_{2 n}\left(\mathcal{O}_{v}\right)_{l}=0$. 


\section{THE CASE OF ODD INDICES}

Observe that by [DF, Theorem 8.7] and [So2, Theorem 1, p. 375] we have the following isomorphism:

$$
K_{2 n+1}(\mathcal{O}[1 / l]) \otimes \mathbb{Z}_{l} / \text { torsion } \rightarrow K_{2 n+1}^{e t}(\mathcal{O}[1 / l]) / \text { tor sion } .
$$

This implies that we have the following surjection:

$$
K_{2 n+1}(\mathcal{O}[1 / l])_{l} \rightarrow K_{2 n+1}^{e t}(\mathcal{O}[1 / l])_{l}
$$

Assume that $v$ does not divide $l$. Consider the following commutative diagram:

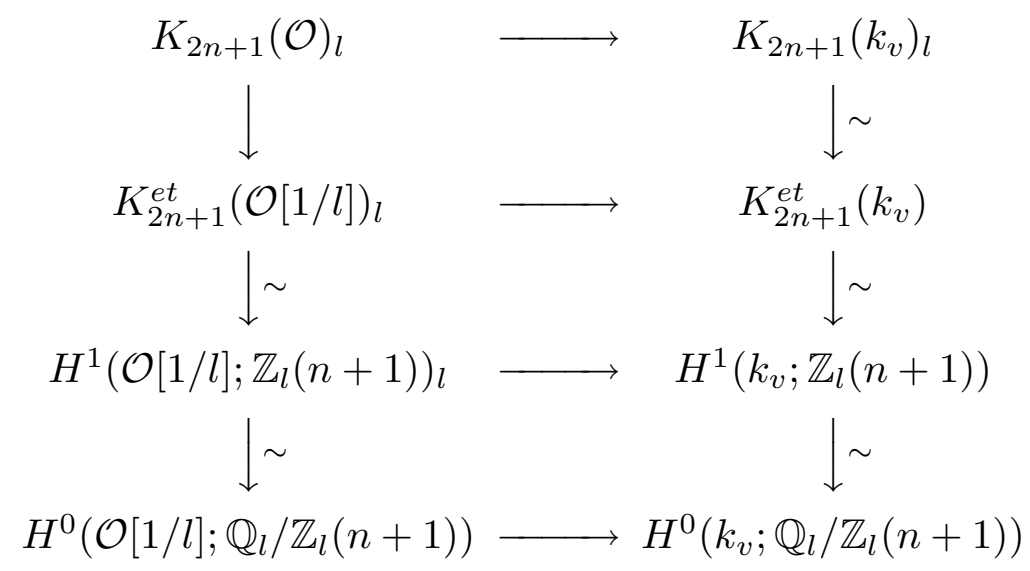

It is easy to see that the bottom horizontal arrow in the diagram (3.2) is an imbedding for all $v$ which do not divide $l$. This shows that the kernel of the upper horizontal arrow is equal to the kernel of the upper, left vertical arrow. Hence, we can conclude the following result.

Lemma 3.3. The Quillen-Lichtenbaum conjecture holds for $K_{2 n+1}(\mathcal{O})$ if and only if the reduction map:

$$
\pi: K_{2 n+1}(\mathcal{O})_{l} \rightarrow K_{2 n+1}\left(k_{v}\right)
$$

is injective for some $v$ which does not divide l. The Quillen-Lichtenbaum conjecture for $K_{2 n+1}(\mathcal{O})$ is also equivalent to the injectivity of the map:

$$
\pi: K_{2 n+1}(\mathcal{O})_{l} \rightarrow \prod_{v} K_{2 n+1}\left(k_{v}\right)
$$

Let us consider the following diagram:

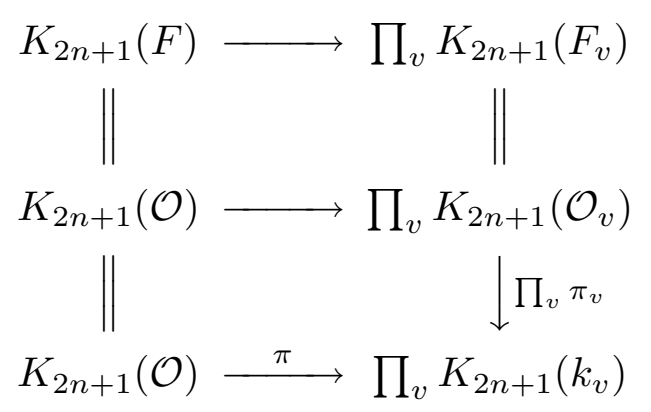

By the lemma 3.3, the diagram (3.4) and the isomorphism (2.3) we obtain the following proposition. 
Proposition 3.5. The Quillen-Lichtenbaum conjecture holds for $K_{2 n+1}, n>0$, if and only if the following localization map:

$$
K_{2 n+1}(F)_{l} \rightarrow \prod_{v} K_{2 n+1}\left(F_{v}\right)_{l}
$$

is injective.

\section{THE CASE OF EVEN INDICES}

Consider the following commutative diagram.

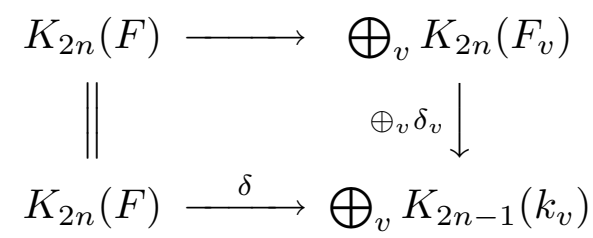

The maps $\delta$ and $\delta_{v}$ come from Quillen localization sequences in K-theory [Q1]. We can write $\bigoplus_{v}$ instead of $\prod_{v}$ for the upper horizontal arrow in diagram (4.1), because of the isomorphism (2.5). The diagram shows that:

$$
W K_{2 n}(F) \subseteq K_{2 n}(\mathcal{O})
$$

The wild kernel $K_{2 n}^{w}(\mathcal{O})_{l}$ was defined by (1.1) cf. [B, p. 288]. At the moment we can not compare the groups $K_{2 n}^{w}\left(\mathcal{O}_{F}\right)_{l}$ and $W K_{2 n}(F)_{l}$, since we do not know the groups $K_{n}\left(\mathcal{O}_{v} ; \mathbb{Z} / l^{k}\right)$ for $v$ dividing $l$ except for the case when $F=\mathbb{Q}$ which is known by results of $[\mathrm{BM}]$. Observe that the definition of the group $W K_{n}(F)$ makes sense for every $l$, including $l=2$ and that for $n=2$ it agrees with the definition of the classical wild kernel for the functor $K_{2}$. By [DF, theorem 8.5, p.275] and by Bockstein sequences we obtain the following natural surjection:

$$
K_{2 n}\left(F_{v}\right)_{l} \rightarrow K_{2 n}^{e t}\left(F_{v}\right)_{l}
$$

for all $v$. This surjection is an isomorphism for every $v$ which does not divide $l$, as we can easily check using results of [Su1] which have been already mentioned. In addition, due to [DF, Prop. 5.1, p.260] and [T, prop. 2.3] we have natural isomorphisms:

$$
K_{2 n}^{e t}\left(F_{v}\right)_{l} \cong H^{2}\left(F_{v} ; \mathbb{Z}_{l}(n+1)\right)_{l} \cong H^{1}\left(F_{v} ; \mathbb{Q}_{l} / \mathbb{Z}_{l}(n+1)\right) / \text { Div. }
$$

Let $D_{n+1}(F)$ denote the group of divisible elements in $H^{2}\left(F ; \mathbb{Z}_{l}(n+1)\right)_{l}$ cf. [Sch, p. 192]. Consider the following commutative diagram.

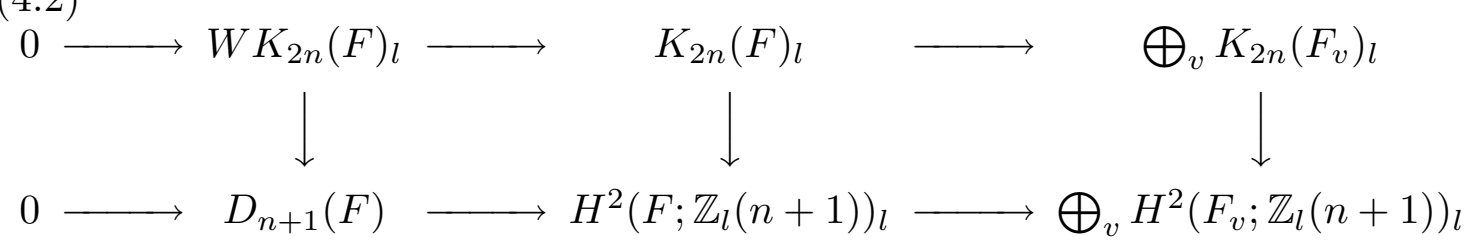


Lemma 4.3. Assume that the natural maps:

$$
K_{2 n}\left(F_{v}\right)_{l} \rightarrow K_{2 n}^{e t}\left(F_{v}\right)_{l}
$$

are isomorphisms for all $v$ which dividel. Then the Quillen-Lichtenbaum conjecture for $K_{2 n}, n>0$, is equivalent to the statement that the left vertical arrow in the diagram (4.2) is an isomorphism.

Proof. Observe that by our assumption the right vertical arrow in (4.2) is an isomorphism. Simple diagram chasing in the diagram (4.2) shows that the left vertical arrow is an isomorphism if and only if the middle vertical arrow is an isomorphism. Hence, our lemma follows by [B, remark 7, p. 295].

\section{Comparison OF CONJECTURES}

The diagrams (3.4), (4.1) and [AB, Theorem 1] give us the following statement.

Proposition 5.1. For each $n \geq 0$ :

$$
W K_{n}(F) \subseteq K_{n}(\mathcal{O})_{t o r}
$$

hence the group $W K_{n}(F)$ is always finite.

Our computations of the previous sections suggest that we should expect that the following conjecture holds true.

Conjecture B. Up to 2-torsion:

$$
W K_{n}(F)=\operatorname{div} K_{n}(F)
$$

for any $n$ and for any number field $F$.

\section{Theorem 5.2.}

(a) Assume that the following maps:

$$
K_{2 m}\left(F_{v}\right)_{l} \rightarrow K_{2 m}^{e t}\left(F_{v}\right)_{l}
$$

are isomorphisms for all $v$ which divide $l$ and for all $m>0$. Then the Quillen-Lichtenbaum conjecture for $F$ is equivalent to the conjecture $B$ for all indices $n>1$.

(b) If $F=\mathbb{Q}$, then the Quillen-Lichtenbaum conjecture and conjecture $B$ are equivalent for $n>1$.

Proof. a)

We observe that $K_{2 n+1}(F)=K_{2 n+1}(\mathcal{O})$ for $n>0$ due to [Q1, Theorem 5] and [So1, Theoreme 3, p. 274]. In addition, $K_{2 n+1}(\mathcal{O})$ is a finitely generated abelian group [Q2, Theorem 1, p. 179]. Hence $\operatorname{div} K_{2 n+1}(F)=0$ for $n \geq 0$. Note that $K_{1}(F)=F^{\times}$[Mi, p. 28] so $\operatorname{div} K_{1}(F)=0$. So the theorem follows by proposition 3.5 and lemma 4.3 .

b) By the results of Bökstedt and Madsen [BM, p. 29], see also [HM, p. 30] the assumption of point (a) holds true for $F=\mathbb{Q}$. 
Proposition 5.3. For every odd prime l we have:

$$
\begin{gathered}
\operatorname{div} K_{n}(\mathbb{Q})_{l} \subset W K_{n}(\mathbb{Q})_{l} \quad \text { for } n \geq 0 \\
K_{2 n}^{w}(\mathbb{Z})_{l}=W K_{2 n}(\mathbb{Q})_{l} \quad \text { for } n>0 .
\end{gathered}
$$

Proof. Let us first prove the first statement. For $F=\mathbb{Q}$ and $n$ even, the right vertical arrow in diagram 4.2 is an isomorphism. In addition the group $H^{2}\left(F_{v} ; \mathbb{Z}_{l}(n+1)\right)_{l}$ is finite [Sch, Satz 4(i) p. 188] for every $l$ odd and every finite prime $v$ of $F$. Hence, the group $\operatorname{div} K_{2 n}(\mathbb{Q})_{l}$ is contained in the kernel of the upper, right horizontal arrow of diagram 4.2. For $n$ odd the first statement is trivial since $\operatorname{div} K_{2 n+1}(F)=0$ for all $n \geq 0$. The second statement follows from diagram 4.2 and the definition of $K_{2 n}^{w}(\mathbb{Z})_{l}$ cf. [B].

Remark 5.4. It follows by [B, theorem 3, p. 289] that:

$$
\operatorname{div} K_{22}(\mathbb{Z})_{\text {odd }}=\mathbb{Z} / 691
$$

In addition we have:

$$
\operatorname{div} K_{22}(\mathbb{Z})_{l} \subset W K_{22}(\mathbb{Q})_{l} \subset K_{22}(\mathbb{Z})_{l}
$$

Let us use the isomorphism $K_{22}(\mathbb{Z})=S K_{1}\left(\mathbb{Z}_{22}\right)$. We can find a matrix $A \in S L\left(\mathbb{Z}_{22}\right)$ corresponding to an element of order 691 in $\operatorname{div} K_{22}(\mathbb{Z})$, such that its inverse can be found by the standard formula involving adjoint matrix, but we cannot find the inverse of $A$ using Gauss-Jordan algorithm. In addition let us consider $A$ as a matrix in $S L\left(\left(\mathbb{Z}_{l}\right)_{22}\right)$. Then by proposition 5.3 we are able to find the inverse of $A$ using Gauss-Jordan algorithm in $S L\left(\left(\mathbb{Z}_{l}\right)_{22}\right)$ for every $l$.

Theorem 5.5. Conjecture $B$ holds true for all number fields $F$ and for $0 \leq n \leq 3$. In addition, it holds true for $n=4$ and $F=\mathbb{Q}$.

Proof. It is obvious for $n=0$ and $n=1$. For $n=2$ it was proven by Tate cf. [Ba, p.250]. For $n=3$ this is a consequence of the well-known result of Merkurjev and Suslin [MS, p.342] which verifies the Quillen-Lichtenbaum conjecture for $K_{3}$, so we can see that our conjecture B holds in this case as follows by the diagram (3.2). By $[\mathrm{RW}]$ we know that $K_{4}(\mathbb{Z})=0$. Hence, div $K_{4}(\mathbb{Q})=0$ and $W K_{4}(\mathbb{Q})=0$ as subgroups of $K_{4}(\mathbb{Z})$.

\section{Conjecture B And matrices}

In this section, for the convenience of the reader, we enclose some results from the paper [BZ2]. Let $R$ denote any commutative ring with identity. For $n>0$ let us denote by $R_{n}$ the ring:

$$
R\left[t_{1}, t_{2}, \ldots, t_{n}\right] /\left(\left(t_{1}^{2}-t_{1}\right) \ldots\left(t_{n}^{2}-t_{n}\right)\right) .
$$

For $n=0$ we put $R_{0}=R$. The ring $R_{n}$ is called the square ring. To simplify notation we put $T=\left(t_{1}, t_{2}, \ldots, t_{n}\right)$ and

$$
\alpha(T)=\left(t_{1}^{2}-t_{1}\right) \ldots\left(t_{n}^{2}-t_{n}\right) .
$$

Observe that:

$$
\alpha(T)=t_{1}^{2} t_{2}^{2} \ldots t_{n}^{2}+(\text { terms of total degree smaller than } 2 n) .
$$


Lemma 6.1. [BZ2, Lemma 1] If $R \subseteq S$ are two commutative rings with identity, then the induced map $R_{n} \rightarrow S_{n}$ is an imbedding.

Lemma 6.2. [DW, Corollary 5.3] Let $R$ be a domain. Then $R_{n}^{*}=R^{*}$ for $n>1$ and $R_{1}^{*}=R^{*} \oplus R^{*}$, where by the upper star we denote the group of units.

Remark 6.3. Let $R$ be a commutative, regular ring with identity. Then by [DW, Corollary 5.4] or by [Su2, p. 351], we have for $n>0$ :

$$
K_{n}(R)=K_{1}\left(R_{n}\right) / K_{1}(R)
$$

Remark 6.4. Let $R$ be a field, the ring of integers in a number field or a local ring. Then $K_{1}(R)=R^{*}$ [Mi, p. 28]. Hence, if $R$ is a field or the ring of integers in a number field or $R$ is a regular, local ring which is a domain, then by the remark 6.3 and lemma 6.2 we get:

$$
K_{n}(R)=K_{1}\left(R_{n}\right) / K_{1}(R)=\left(S K_{1}\left(R_{n}\right) \oplus R^{*}\right) / R^{*}=S K_{1}\left(R_{n}\right)
$$

cf. [DW, Corollary 5.3]. In particular, the definition of the group $W K_{n}(F)$ can be rewritten as the following exact sequence.

$$
0 \rightarrow W K_{n}(F) \rightarrow S K_{1}\left(F_{n}\right) \rightarrow \prod_{v} S K_{1}\left(F_{v n}\right)
$$

Remark 6.5. Let $A \in S L\left(F_{n}\right)$. Let $[A]$ denote the class of the matrix $A$ in the group $S K_{1}\left(F_{n}\right)_{\text {tor }}$. Then $[A] \in W K_{n}(F)$ if and only if $A$ is row- or column-equivalent or both row- and column-equivalent to the identity matrix in $S L\left(F_{v n}\right)$ for each $v$.

It is easy to show that in the case of even indices we have the following exact sequence.

$$
0 \rightarrow W K_{2 n}(F) \rightarrow K_{2 n}(\mathcal{O}[1 / l]) \rightarrow \prod_{v \mid l} K_{2 n}\left(F_{v}\right)
$$

Remark 6.6. Comparison of the local and global localization sequences shows that we have the following exact sequence for all $n>0$.

$$
0 \rightarrow W K_{n}(F) \rightarrow S K_{1}\left(\mathcal{O}_{n}\right) \rightarrow \prod_{v} S K_{1}\left(\mathcal{O}_{v n}\right)
$$

It was proven in $[\mathrm{BZ2}]$ that the natural maps:

$$
\begin{aligned}
& K_{n}\left(\mathcal{O}_{v}^{h}\right) \rightarrow K_{n}\left(\mathcal{O}_{v}\right) \\
& K_{n}\left(F_{v}^{h}\right) \rightarrow K_{n}\left(F_{v}\right)
\end{aligned}
$$

are injective. Hence, we obtain the following exact sequences.

$$
0 \rightarrow W K_{n}(F) \rightarrow S K_{1}\left(F_{n}\right) \rightarrow \prod_{v} S K_{1}\left(F_{v n}^{h}\right)
$$




$$
0 \rightarrow W K_{n}(F) \rightarrow S K_{1}\left(\mathcal{O}_{n}\right) \rightarrow \prod_{v} S K_{1}\left(\mathcal{O}_{v n}^{h}\right)
$$

Observe that for any field extension $K \subset L$ we have the following exact sequence.

$$
0 \rightarrow S L\left(K_{n}\right) \cap E\left(L_{n}\right) / E\left(K_{n}\right) \rightarrow S K_{1}\left(K_{n}\right) \rightarrow S K_{1}\left(L_{n}\right)
$$

From (6.7) and (6.8) we obtain the following equality.

$$
W K_{n}(F)=\left(S L\left(F_{n}\right) \cap \bigcap_{v} E\left(F_{v n}^{h}\right)\right) / E\left(F_{n}\right)=\left(S L\left(\mathcal{O}_{n}\right) \cap \bigcap_{v} E\left(\mathcal{O}_{v n}^{h}\right)\right) / E\left(\mathcal{O}_{n}\right)
$$

Remark 6.9. Observe that the conjecture B says that for each odd prime $l$ we should have:

$$
W K_{n}(F)_{l}=\bigcap_{r \geq 1}\left(S K_{1}\left(F_{n}\right)_{l}\right)^{r} .
$$

We can also look at the question of descent using the $S K_{1}$ description of $K$ theory. Let $L / K$ be a Galois extension with the Galois group $G=G(L / K)$.

Proposition 6.10. The natural map:

$$
K_{n} \stackrel{\sim}{\longrightarrow} L_{n}^{G}
$$

is an isomorphism.

Proof. Consider the following commutative diagram.

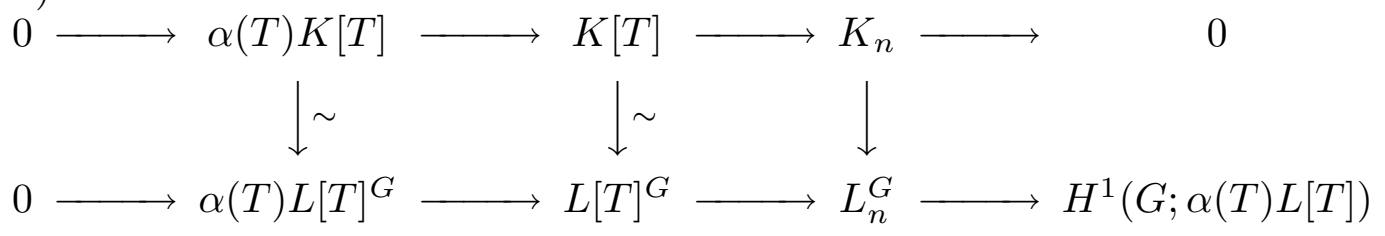

We observe that:

$$
\alpha(T) L[T] \stackrel{\sim}{\longrightarrow} \bigoplus L
$$

as $G$-modules, where the direct sum is over all monomials $t_{1}^{r_{1}} t_{2}^{r_{2}} \ldots t_{n}^{r_{n}}$ of $L[T]$ for $r_{i} \geq 0$. By $\left[\mathrm{CF}\right.$, chapter V.2.6, Proposition 2.1] we know that $H^{1}(G ; L)=0$. Hence:

$$
H^{1}(G ; \alpha(T) L[T])=H^{1}(G ; \bigoplus L)=\bigoplus H^{1}(G ; L)=0
$$

and the proposition follows from the five lemma applied to the diagram (6.11). 
Corollary 6.12. The isomorphism from proposition 6.10 induces the following isomorphism.

$$
S L\left(K_{n}\right) \stackrel{\sim}{\longrightarrow} S L\left(L_{n}\right)^{G}
$$

Remark 6.13. Observe that we only have $E\left(K_{n}\right) \subseteq E\left(L_{n}\right)^{G}$. Consider the following commutative diagram.

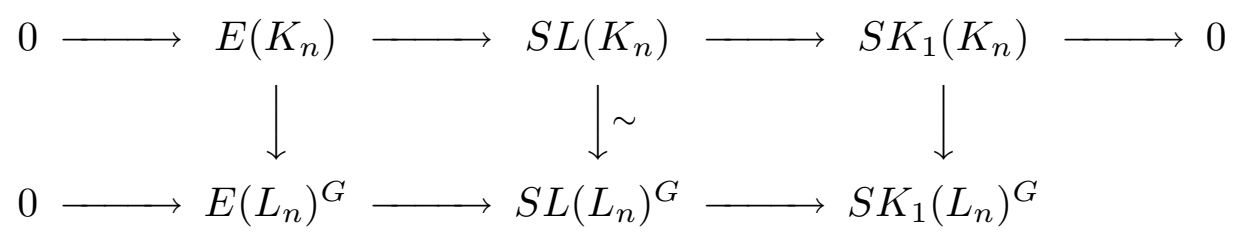

Remark 6.15. The diagram (6.14) implies that we have the following exact sequence.

$0 \rightarrow S L\left(K_{n}\right) \cap E\left(L_{n}\right) / E\left(K_{n}\right) \rightarrow K_{n}(K) \rightarrow K_{n}(L)^{G} \rightarrow S K_{1}\left(L_{n}\right)^{G} / \operatorname{Im} S L\left(K_{n}\right) \rightarrow 0$

7. Conditions for triviality of Elements in $W K_{n}(F)$

Let $R$ be a commutative ring with identity. Let $A \in E(R)$ and let us write $A=\prod_{i, j, \lambda} e_{i, j}^{\lambda}$ in terms of elementary matrices.

Lemma 7.1. Let $A$ be a product of elementary matrices $e_{i, j}^{\lambda}$ that are pairwise commutative. Then all entries of the matrix $A$ which are on the main diagonal are equal to 1 and all other entries are linear homogeneous polynomials in $\lambda$ 's. The polynomials have integral coefficients.

Proof. The proof is by induction on the number of elementary matrices. It is obvious for one such a matrix. Let $B=\left(b_{i j}\right)_{1 \leq i, j \leq m} \in G L(R)$ which commutes with elementary matrices $e_{i, j}^{\lambda} \in E(R)$. For our computations we assume that $i<j$. The case $i>j$ is similar. Consider products of matrices:

$$
B e_{i, j}^{\lambda}=\left(\begin{array}{ccccccc}
b_{11} & b_{12} & \ldots & b_{1 j-1} & b_{1 j}+\lambda b_{1 i} & \ldots & b_{1 m} \\
b_{21} & b_{22} & \ldots & b_{2 j-1} & b_{2 j}+\lambda b_{2 i} & \ldots & b_{2 m} \\
\vdots & \vdots & \vdots & \vdots & \vdots & \vdots & \vdots \\
b_{m 1} & b_{m 2} & \ldots & b_{m j-1} & b_{m j}+\lambda b_{m i} & \ldots & b_{m m}
\end{array}\right)
$$

On the other hand:

$$
e_{i, j}^{\lambda} B=\left(\begin{array}{cccc}
b_{11} & b_{12} & \ldots & b_{1 m} \\
b_{21} & b_{22} & \ldots & b_{2 m} \\
\vdots & \vdots & \vdots & \vdots \\
b_{(i-1) 1} & b_{(i-1) 2} & \ldots & b_{(i-1) m} \\
\vdots & \vdots & \vdots & \vdots \\
b_{i 1}+\lambda b_{j 1} & b_{i 2}+\lambda b_{j 2} & \ldots & b_{i m}+\lambda b_{j m} \\
\vdots & \vdots & \vdots & \vdots \\
b_{m 1} & b_{m 2} & \ldots & b_{m m}
\end{array}\right)
$$


Comparison of entries gives: $b_{k j}+\lambda b_{k i}=b_{k j}$ for $k \neq i$. So, $\lambda b_{k i}=0$. In the same way $b_{i l}+\lambda b_{j l}=b_{i l}$ for $l \neq j$ and $\lambda b_{j l}=0$. At the end we compare the $i j$ entries: $b_{i j}+\lambda b_{i i}=b_{i j}+\lambda b_{j j}$. This implies that $\lambda\left(b_{i i}-b_{j j}\right)=0$. So in particular if $b_{i i}=b_{j j}$, the $i j$ entries of the matrices in question are equal. To continue observe that if $A=B e_{i, j}^{\lambda}$, where $B$ is a product of pairwise commuting elementary matrices, then by inductive hypothesis $B$ is a matrix with 1 's on the main diagonal and the other entries are linear homogeneous polynomials od $\lambda$ 's. Let us denote $a_{i j}$ the entries of $A$. Hence the above comparison of entries shows that all the entries of $A$ on the main diagonal are equal to 1 . It is so because $a_{r r}=b_{r r}=1$ for $r \neq j$. In addition $a_{j j}=b_{j j}+\lambda b_{j i}=b_{j j}=1$. Also all entries of $A$ beyond the $j$-th column are just the same as the corresponding entries of $B$. Using again the comparison of entries due to the equation $B e_{i, j}^{\lambda}=e_{i, j}^{\lambda} B$ we see that in the $j$-th column $a_{k j}=b_{k j}+\lambda b_{k i}=b_{k j}$ for $k \neq i$ so this case follows by inductive hypothesis. On the other hand for $k=i$ we have $a_{i j}=b_{i j}+\lambda b_{i i}=b_{i j}+\lambda$ because $b_{i i}=1$ by the induction.

Proposition 7.2. Let $A \in S K_{1}\left(F_{n}\right)$. Assume that for some finite prime $v, A$ can be written as a product of elementary, pairwise commuting matrices in $S L_{1}\left(F_{v n}^{h}\right)$. Then $A$ determines a divisible element in $S K_{1}\left(F_{n}\right)$.

Proof.. Let $A=\prod_{i, j, \lambda} e_{i, j}^{\lambda}$ in $S L_{1}\left(F_{v n}^{h}\right)$ be a product of elementary, pairwise commutative matrices. For any natural number $m$ consider the matrix $C=\prod_{i, j, \lambda} e_{i, j}^{\lambda / m}$. The matrices of the form $e_{i, j}^{\lambda / m}$ also commute pairwise. Hence by lemma 8.1 we see that $C$ comes from an element of $S L\left(F_{n}\right)$. Let us denote this element $C_{0}$. But $C_{0}^{m}=C^{m}=A$ in $S L\left(F_{v n}^{h}\right)$. Hence by lemma 6.1 we get $C_{0}^{m}=A$ in $S L\left(F_{n}\right)$.

Corollary 7.3. Let $A \in S K_{1}\left(F_{n}\right)$ be an element of $W K_{n}(F)$. Assume that for some finite prime $v, A$ can be written as a product of elementary, pairwise commuting matrices in $S L\left(F_{v n}^{h}\right)$. Then $A$ determines a divisible element in $S K_{1}\left(F_{n}\right)$.

Remark 7.4. Let $A \in S L\left(F_{n}\right)$ be a matrix whose class $\bmod E\left(F_{n}\right)$ is in $W K_{n}(F)_{l}$. Proposition 7.2 and corollary 7.3 show, that if a matrix $A$ is a product of pairwise commutative matrices in $S L\left(F_{v n}^{h}\right)$, for some $v$, then this matrix $A$ gives a trivial class in $S K_{1}\left(F_{n}\right)$. Indeed, computations in proposition 7.2 and corollary 7.3 show that for each power $l^{k}$ of an odd prime number $l$ we can find a matrix $B_{k} \in S L\left(F_{n}\right)$ such that $A=B_{k}^{l^{k}}$. Observe that actually $A \in S L\left(\mathcal{O}_{S, n}\right)$ for some finite set $S$ of prime numbers containing $l$ and $B \in S L\left(\mathcal{O}_{S, n}\right)$ by computations. Hence the class of $A$ is divisible by all powers of $l$ in a finite group $K_{n}\left(\mathcal{O}_{S}\right)_{\text {tor }}$. Hence, the class of $A$ must be trivial. This also follows from the corollary 7.6 below.

Let us note first that every elementary matrix with coefficients in any commutative ring can be written as a product of at most four unipotent matrices:

$$
U_{1} L_{1} U_{2} L_{2} \quad \text { or } \quad L_{1} U_{1} L_{2} U_{2}
$$

where $U_{i}\left(L_{i}\right.$, respectively) denote upper (lower, resp.) triangular matrix with 1 on the diagonal cf. [vdK, LNM 966, p.358]. Conversely every upper or lower triangular matrix with 1 on the diagonal is clearly a product of elementary matrices, since we can bring such a matrix to the identity matrix by a series of elementary operations. 
Proposition 7.5. Let $R$ be a ring contained as a subring in $S$. Let $A$ be a matrix in $S L(R)$ such that it is a product of at most two unipotent triangular matrices with coefficients in $S$. Then the unipotent matrices have coefficients in $R$.

Proof. The case when $A$ is a unipotent matrix is obvious. Let $A$ be a product of two unipotent matrices: $A=U L$, where $U$ is upper triangular and $L$ is lower triangular. The case when $A=L U$ is handled similarly. Let us put:

$$
\begin{gathered}
U=\left(\begin{array}{cccccccc}
1 & u_{12} & u_{13} & \ldots & \ldots & \ldots & \ldots & u_{1 m} \\
0 & 1 & u_{23} & \ldots & \ldots & \ldots & \ldots & u_{2 m} \\
\vdots & \vdots & \vdots & \vdots & \vdots & \vdots & \vdots & \vdots \\
0 & 0 & \ldots & 1 & \ldots & u_{i(i+1)} & \ldots & u_{i m} \\
\vdots & \vdots & \vdots & \vdots & \vdots & \vdots & \vdots & \vdots \\
0 & 0 & & & \ldots & & & 1
\end{array}\right) \\
L=\left(\begin{array}{cccccccc}
1 & 0 & 0 & & \ldots & & & 0 \\
l_{21} & 1 & 0 & & \ldots & & & 0 \\
\vdots & \vdots & \vdots & \vdots & \vdots & \vdots & \vdots & \vdots \\
l_{j 1} & \ldots & l_{j(j-1)} & 1 & \ldots & \ldots & 0 & 0 \\
\vdots & \vdots & \vdots & \vdots & \vdots & \vdots & \vdots & \vdots \\
l_{m 1} & l_{m 2} & \ldots & \ldots & \ldots & \ldots & l_{m(m-1)} & 1
\end{array}\right) .
\end{gathered}
$$

The product $U L$ is the matrix $A$ with coefficients in $R$. Observe that multiplying rows of $U$ by last column of $L$ we can figure out that last column of $U$ has coefficients in $R$. Also multiplying last row of $U$ by columns of $L$ we see that last row of $L$ has coefficients in $R$. Now the argument goes by induction. Assume that last $k$ columns of $U$ and last $k$ rows of $L$ are already in $R$. Multiplying the $m-k$-th row of $U$ by $j$-th column of $L$ for $j<m-k$ we get:

$$
l_{(m-k) j}+\sum_{i=m-k+1}^{m} u_{(m-k) i} l_{i j} \in R .
$$

Hence, by the induction hypotheses $l_{(m-k) j} \in R$ for all $j<m-k$. In the same way, multiplying $i$-th row of $U$ for $i<m-k$ by $m-k$-th column of $L$ we get:

$$
u_{i(m-k)}+\sum_{j=m-k+1}^{m} u_{i j} l_{j(m-k)} \in R
$$

Hence, by the induction hypotheses $u_{i(m-k)} \in R$ for all $i<m-k$. We proved that last $k+1$ columns of $U$ and last $k+1$ rows of $L$ are in $R$. The claim follows by induction.

Corollary 7.6. Let $A \in S L\left(F_{n}\right)$ be a matrix whose class modulo $E\left(F_{n}\right)$ is in $W K_{n}(F)$. Let for some $v$ the matrix $A$ be a product in $S L\left(F_{v n}\right)$ (or $S L\left(F_{v n}^{h}\right)$ ) of at most two unipotent triangular matrices or $A$ be a product of four unipotent triangular matrices such that the first and fourth factors of this product have coefficients in $F_{n}$. Then the class of $A$ is trivial in $W K_{n}(F)$.

Proof. Follows immediately from propostion 7.5. 
Remark 7.7. A unipotent triangular matrix from $S L\left(F_{v n}\right)$ can be written in the form $I+N$ where $N$ is an upper or lower triangular with zero's on the main diagonal. Coefficients of $N$ are elements of $F_{v n}$ so for each natural number $k$ every such coefficient can be written as a sum of an element of $F_{n}$ and an element from $\left(\pi_{v}\right)^{k} \mathcal{O}_{v n}$, where $\pi_{v}$ is a uniformizer of the maximal ideal of $\mathcal{O}_{v}$. Hence, we can write:

$$
I+N=\left(I+N_{0}\right)\left(I+\left(\pi_{v}\right)^{k} N_{1}\right) \quad \text { or } \quad I+N=\left(I+\left(\pi_{v}\right)^{k} N_{1}^{\prime}\right)\left(I+N_{0}\right),
$$

where $N_{0}$ and $N_{1}$ are upper (lower) triangular and nilpotent. $N_{0}$ has coefficients in $F_{n}$ and $N_{1}, N_{1}^{\prime}$ have coefficients in $\mathcal{O}_{v n}$.

Proposition 7.8. Let $[A] \in W K_{n}(F)$. For each prime $v$ there is a matrix $B \in$ $S L\left(\mathcal{O}_{n}\right)$ such that $[A]=[B]$ in $W K_{n}(F)$ and:

$$
B \equiv I \bmod v
$$

Proof. Let $A \in S L\left(\mathcal{O}_{n}\right)$ be a matrix whose class modulo $E\left(\mathcal{O}_{n}\right)$ is in $W K_{n}(F)$. Any element in $W K_{n}(F)$ can be represented in such a way by the exact sequence (6.8). Pick a prime ideal $v$ and write:

$$
A=U_{1} L_{1} U_{2} L_{2}
$$

where the matrices in the product have coefficents in $\mathcal{O}_{v n}$. The case when the product on the right of the last matrix equation starts with a lower triangular matrix is analogous. By remark 7.7 we can write:

$$
U_{1}=U_{1}^{\prime} U_{1}^{\prime \prime} \quad \text { and } \quad L_{2}=L_{2}^{\prime \prime} L_{2}^{\prime},
$$

where $U_{1}^{\prime}$ is a unipotent matrix with coefficients above main diagonal in the ring $\mathcal{O}_{n}$ and $U_{1}^{\prime \prime}$ is an unipotent matrix with coefficients above the main diagonal in $\left(\pi_{v}\right)^{k} \mathcal{O}_{v n}$. Similarly, $L_{2}^{\prime}$ is an unipotent matrix with coefficients below the main diagonal in $\mathcal{O}_{n}$ and $L_{2}^{\prime \prime}$ is a unipotent matrix with coefficients below the main diagonal in $\left(\pi_{v}\right)^{k} \mathcal{O}_{v n}$. Hence, we get:

$$
U_{1}^{\prime-1} A L_{2}^{\prime-1}=U_{1}^{\prime \prime} L_{1} U_{2} L_{2}^{\prime \prime} .
$$

Matrices $A$ and $B=U_{1}^{\prime-1} A L_{2}^{\prime-1}$ are in the same class modulo $E\left(\mathcal{O}_{n}\right)$. Again by remark 7.7 we can also write:

$$
L_{1}=L_{1}^{\prime \prime} L_{1}^{\prime} \quad \text { and } \quad U_{2}=U_{2}^{\prime} U_{2}^{\prime \prime},
$$

where $U_{2}^{\prime}$ is a unipotent matrix with coefficients above main diagonal in the ring $\mathcal{O}_{n}$ and $U_{2}^{\prime \prime}$ is an unipotent matrix with coefficients above the main diagonal in $\left(\pi_{v}\right)^{k} \mathcal{O}_{v n}$. Similarly, $L_{1}^{\prime}$ is an unipotent matrix with coefficients below the main diagonal in $\mathcal{O}_{n}$ and $L_{1}^{\prime \prime}$ is a unipotent matrix with coefficients below the main diagonal in $\left(\pi_{v}\right)^{k} \mathcal{O}_{v n}$. We have the following equality:

$$
L_{1}^{\prime-1} U_{1}^{\prime-1} A L_{2}^{\prime-1} U_{2}^{\prime-1}=L_{1}^{\prime-1} U_{1}^{\prime \prime} L_{1} U_{2} L_{2}^{\prime \prime} U_{2}^{\prime-1} .
$$

Matrices $A, A_{1}=U_{1}^{\prime-1} A L_{2}^{\prime-1}$ and $A_{2}=L_{1}^{\prime-1} U_{1}^{\prime-1} A L_{2}^{\prime-1} U_{2}^{\prime-1}$ are in the same class modulo $E\left(\mathcal{O}_{n}\right)$. It is clear from (7.9) that:

$$
A_{2} \equiv I \bmod v
$$




\section{REFERENCES}

[AB] D.Arlettaz and G.Banaszak, On the non-torsion elements in the Algebraic K-theory of rings of integers, J. Reine Angew. Math. 461 (1995), 63-79.

[B] G.Banaszak, Generalization of the Moore exact sequence and the wild kernel for higher K-groups, Comp. math. 86. (1993), 281-305.

[BZ1] G.Banaszak and P.Zelewski, Continuous K-theory, K-theory 9 (1995), 379-392.

[BZ2] G.Banaszak and P.Zelewski, On the map between $K$-theory of henselization and completion of some local rings, Journal of Pure and Applied Algebra 120 (1997), 161-165.

[Ba] H.Bass, $K_{2}$ des corps globaux, Séminaire BOURBAKI, $23^{e}$ année $n^{o} 394$ (1970/71).

[CF] J.W.S.Cassels and A.Frohlich, Algebraic Number Theory, Academic Press, 1967.

[BM] M.Bökstedt and I.Madsen, Algebraic K-theory of local number fields; the unramified case. Prospects in Topology. (ed. F.Quinn), Ann. of Math. Stud. 138 (1995), Princeton Univ. Press, 28-57.

[DW] B.H.Dayton and C.A.Weibel, K-theory of hyperplanes, Transactions of the AMS 257, n.1 (1980), 119-141.

[DF] W.Dwyer and E.Friedlander, Algebraic and étale K-theory, Transactions of the AMS 292, n.1 (1985), 247-280.

[HM] L.Hesselholt and I.Madsen, On the K-theory of finite algebras over Witt vectors of perfect fields, Topology 36 (1997), 29-101.

$[\mathrm{vdK}]$ W.van der Kallen, $S L_{3}(\mathbb{C}[x])$ does not have bounded word length, Springer Lecture Notes in Mathematics 966, 357-361.

[MS] A.Merkurjev and A.Suslin, On the $K_{3}$ of a field, Math. USSR Izvestya 36, n.3 (1991), 531-565.

[Mi] J.Milnor, Introduction to Algebraic K-theory, Annals of Mathematics Studies, Princeton University Press, Princeton, N.J., 1971.

[Q1] D. Quillen, Higher Algebraic K-theory I, Lecture Notes in Mathematics 341 (1973), 85147.

[Q2] D. Quillen, Finite generation of the group $K_{i}$ of ring of algebraic integers, Lecture Notes in Mathematics 341 (1973), 179-198.

[RW] J.Rognes and C.Weibel, Two-primary algebraic $K$-theory of rings of integers in number fields, preprint (1997).

[Sch] P. Schneider, Über gewisse Galoiskohomologiegruppen, Math. Zeit. 168 (1979), 181-205.

[So1] C. Soulé, K-theorié de anneaux d'entiers de corps de nombres et cohomologie étale, Inventiones math. 55 (1979), 251-295.

[So2] C. Soulé, On higher p-adic regulators, Lecture Notes in Mathematics, 854 (1981), 372-401.

[Su1] A.Suslin, On the K-theory of local fields, J. of Pure and Applied Algebra 34 (1984), 301318.

[Su2] A.Suslin, Mennicke symbols and their applications in the K-theory of fields, Lecture Notes in Mathematics 966 (1982), 334-356.

[T] J. Tate, Relations between $K_{2}$ and galois cohomology, Inventiones math. 36 (1976), 257274 .

Department of Mathematics, Adam Mickiewicz University, Poznań, Poland E-mail address: BANASZAK@math.amu.edu.pl

Department of Mathematics, Adam Mickiewicz University, Poznań, Poland E-mail address: GAJDA@math.amu.edu.pl

Department of Mathematics, Szczecin University, 70-451 Szczecin, Poland

E-mail address: KRASON@uoo.univ.szczecin.pl

36-11 Pirie Drive, Dundas, Ontario L9H 6X5, Canada

E-mail address: PIOTR@icarus.math.mcmaster.ca 